$17^{\text {th }}$ International Congress of Metrology, 07008 (2015)

DOI: $10.1051 /$ metrology $/ 201507008$

(C) Owned by the authors, published by EDP Sciences, 2015

\title{
Metrology to underpin future regulation of industrial emissions
}

\author{
Anne Rausch ${ }^{1, a}$, Olav Werhahn ${ }^{1, b}$, Oliver Witzel ${ }^{1}$, Volker Ebert ${ }^{1, c}$, Edgar Moreno Vuelban ${ }^{2}$, Jan Gersl ${ }^{3}$, Gjermund \\ Kvernmo ${ }^{4}$, John Korsman ${ }^{5}$, Marc Coleman ${ }^{6}$, Tom Gardiner ${ }^{6}$ and Rod Robinson ${ }^{6}$ \\ ${ }^{1}$ Physikalisch-Technische Bundesanstalt, Bundesallee 100, 38116 Braunschweig, Germany \\ ${ }^{2}$ VSL B.V., Thijsseweg 11, NL-2629 JA Delft, Netherlands \\ ${ }^{3}$ Cesky Metrologicky Institut Brno, Okružni 31, CZ-638 00 Brno, Czech Republic \\ ${ }^{4}$ Justervesenet, Fetveien 99, NO-2007 Kjeller, Norway \\ ${ }^{5}$ DCMR Environmental Protection Agency, Parallelweg 1, 3112 NA, Schiedam, Netherlands \\ ${ }^{6}$ National Physical Laboratory, Hampton Road, Teddington, Middlesex, TW11 OLW, United Kingdom
}

\begin{abstract}
Monitoring industrial pollutant emissions to air to ensure compliance with EU directives and national legislation is the key to enforcing emission limits and thereby enabling their reduction and control. Industry needs to measure and report emissions for regulatory purposes including assessing stack emissions against concentration limit values, reporting annual mass emissions, and determining emissions of GHGs from area sources. Industry and regulators require a robust metrology infrastructure to underpin the monitoring and reporting framework. The EMRP project IMPRESS [1] will address many of the current technological obstacles and enhance the reporting and therefore the control of industrial emissions within the framework of increasingly lower limit values. IMPRESS targets measurement and monitoring technologies, methodologies and guidance to support industry and regulators as well as new CEN standards.
\end{abstract}

\section{The European level}

Accurate measurement of emissions of pollutants and greenhouse gasses (GHGs) to the atmosphere is vital in enabling action to control and reduce air pollution in order to protect European citizens and the environment. The European Climate Change Programme (ECCP) is targeting an $80 \%-95 \%$ reduction in emissions by 2050 compared to 1990 levels. However, current evidence shows that emissions are only likely to be reduced to $30 \%$ by 2030 . Hence we are not on track and more stringent emission limits will be necessary as will measurement methods to enable monitoring and enforcement.

The emission of pollutants from industrial sources has a direct impact on air quality. As stated by the European Environment Agency EEA, the EU's long-term objective is to achieve levels of air quality that do not result in unacceptable impacts on, and risks to, human health and the environment. These harmful emissions also have an economic impact, the EEA state on their website that the cost of air pollution from the 10,000 largest polluting facilities in Europe was $€ 102$ to $€ 169$ billion in 2009 .

Current regulation (EU Industrial Emissions Directive (IED) and Best Available Technology Reference (BREF) documents are introducing lower emission limit values, in some cases requiring measurements not achievable by current standard methods, and novel reporting requirements - for example annual mass emission values for emissions trading and the quantification of emissions from area sources (e.g. fugitive leaks of VOCs from industrial plant and GHGs from landfills). Improved technologies, methods and protocols are required by including industry (e.g. operators in the manufacturing, waste sectors), regulatory authorities, equipment suppliers and stack monitoring providers to enable these lower limits and novel emissions sources to be controlled. Industry and regulators require a robust metrology infrastructure to underpin the monitoring and reporting framework.

\section{The challenge}

EU policies aim to reduce exposure to air pollution by reducing emissions and setting limits and target values for air quality. To achieve this in 2012 the Commission published the Industrial Emissions Directive (IED)[1] (replacing a number of existing directives including the Waste Incineration [2] and Large Combustion Plant [3] directives) bringing into force increasingly stringent emission limit values (ELVs) for key species (SO2, NOx, $\mathrm{HCl}$, etc.). The reporting of industrial emissions is also required under the European Pollutant Release and

\footnotetext{
a Anne Rausch: anne.rausch@ptb.de

b Olav Werhahn: olav.werhahn@ptb.de

${ }^{c}$ Volker Ebert: volker.ebert@ptb.de
} 
Transfer Register (E-PRTR), which requires reporting of total mass emissions of key pollutants.

In addition, the specific reduction in the emission of greenhouse gases GHGs is a key goal in the EU's policy to tackle climate change. The reduction of $\mathrm{CO} 2$ emissions is mainly addressed through the European Emissions Trading Scheme. However, industrial emission of methane to air, a key GHG species, is mainly from area sources such as landfills in the waste sector and fugitive leaks in the energy sector. Industrial emissions arise from both point (e.g. stack) and area (e.g. landfill) sources which present different measurement issues and governing regulation.

For stack emissions the EU requires plant operators to report data which are traceable to Standard Reference Methods (SRMs). The Commission mandated the Comite Européen du Normalisation (CEN) under Technical Committee (TC) 264 to develop SRMs for the species referred to within the IED. However, whilst the regulatory framework is sound most of the SRMs were produced many years ago and there is mounting evidence that they may no longer be suitable for enforcing recent (i.e. IED) and upcoming emission limit values. The required monitoring uncertainty is given in the IED and is expressed as a percentage of emission limit value, hence, as ELVs decrease so do the required uncertainties. For example, the IED stipulates that the monitoring uncertainty requirement for $\mathrm{SO} 2$ is $\pm 10 \%, \mathrm{k}=1$, hence, as the ELV at a waste incinerator is $19 \mathrm{ppm}$ the required uncertainty is $\pm 1.9 \mathrm{ppm}$, whereas recent work has shown that accredited stack monitoring service providers can deviate by as much as $6 \mathrm{ppm}$ [4]. Such uncertainty has a compounding effect since the role of the stack monitoring organisation is two-fold in that in addition to testing for compliance they must also carry out a series of parallel measurements in accordance with EN 14181 [5] in order to calibrate incumbent on-line plant operator installed instrumentation that demonstrates compliance for the remainder of the year. Consequently, the accuracy of all continuous emission monitor (CEM) systems across the EU (also referred to as automated measuring systems AMS) is dependent on the SRM and how well it is followed. The existence of such issues is beginning to be more formerly debated within the community since, at the 22nd CEN plenary meeting on 23/24 May 2012, the issue was raised by several Member States under "Assessment of current SRM to meet stricter limit values" [6].

As stated above, area source emissions are also an important part of the industrial emissions problem. Area sources make a significant contribution to greenhouse gas (GHG) emissions, for example, waste (e.g. landfill) contributes $30 \%$ of all methane emissions across Europe and fugitives (e.g. oil refineries via small leaks in flanges / pipework etc.) a further $19 \%$ [7,8]. Furthermore, area sources are moving even further up the political agenda as many Member States are considering exploiting shale gas reserves, where the public are sure to demand robust reassurance and frequent monitoring to demonstrate negligible environmental impact. One example of mitigation of area source emissions is that the European
Commission is in the process of producing a Best Available Technique Reference (BREF) document and BAT Conclusions to cover the oil and gas industry. However, due to their nature, area sources are difficult to measure and there are no CEN standardised methods for area source emissions quantification and validation data is relatively sparse. Consequently, enforcing BREF / directive requirements in the area sources sector is going to prove very difficult if these issues are not addressed.

With regard to proficiency testing, state of the art in the majority of member states is to send out reference materials in 'blind' testing (e.g. gas cylinders, dust simulants etc.). The key issue here is that this only tests analytical proficiency not sampling proficiency, which is a key element of the SRM. An example of an unresolved issue is with respect to dust measurement. As for gaseous species, dust is monitored by CEM systems (often crossstack optical measurements on the principle of opacity) that must be calibrated in accordance with EN 14181 following the dust SRM, EN 13284-1 [9]. Recent results have shown deviations from scheme participants of up to $50 \%$ of assigned value [10], whereas the SRM stipulated uncertainty in weighing is $2.5 \%, \mathrm{k}=1$. Hence, validated facilities are needed if sampling proficiency is to be measured and if we are to understand if the sampling procedure of an SRM is still fit for purpose

Many CEM systems are not fitted with flow monitors, and there are relatively few continuous flow monitors State of the art in flow measurement is currently represented by the recently developed stack flow methods for continuous and reference measurements developed by CEN TC 264 WG23 (convened by NPL). The validation study for these standards [ref CEN VDI reports] showed there is a significant impact on the flow measurements from the flow conditions in the stack (flow stability, flow inhomogeneity and the flow profile). In addition there were unexplained differences between different measurement techniques, leading to uncertainties that can be higher than the required $2.5 \%$ for annual mass emission data under the emissions trading scheme.

Remote sensing techniques offer an opportunity to address many of the described issues and advance beyond the current state of the art of emissions factors / point measurements, however, as described above, there is a pressing need for standardisation and case studies to provide fully characterised validation data to facilitate uptake.

\section{The project}

The EMRP-project IMPRESS ("Metrology to underpin future regulation of industrial emissions") brings together experts from many European countries combining their years of experience in sensitive measurement techniques and sampling, to provide a comprehensive study on how to monitor industrial emissions now and in the future.

This project aims to enhance the measurement possibilities for emissions of criteria pollutants with lower emission limit values and uncertainties unachievable with current SRMs. Within the project 
protocols are developed and testing is carried out to support the development of techniques with near-future potential to replace existing SRMs with improved capabilities.

After the existing proficiency scheme data is analysed, what will promote a better understanding of the current performance of the European Stack testing industry, the performance of new techniques (e.g. Tuneable Diode Laser Absorption Spectroscopy as described in [12] and ref. therein) to provide improved capabilities beyond current state of the art will be investigated. As well the capabilities of the existing SRMs to meet the stricter requirements now in place will be evaluated and new protocols for improved monitoring methods which will be taken forward into CEN standardisation to extend the range of available methods will be developed. Also national facilities able to test sampling proficiency of SRMs and stack testing organisations will be constructed and validated. These facilities will also extend the state of the art by providing new capabilities within NMIs to support instrument development.

Advancements in flow and mass emission measurements will be achieved by providing enhanced techniques and capabilities to determine measurement uncertainties. To provide new understanding on the sources of uncertainties in typical stack measurement configurations a CFD model to assess uncertainties in flow measurement will be developed. This work will extend the state of the art currently available within the CEN standards. Sophisticated methods for uncertainty analysis for annual mass emission calculations and a model of the annual mass emission determination process (flow and concentration measurements), providing new insight into the sources of uncertainties in reporting mass emissions will be developed and the use of surrogate parameters to improve uncertainties will be tested. Especially the project will focus on a harmonised approach across Europe by providing new methods and guidelines to enable industry to report uncertainties in annual mass emission values.

By means of area sources the project aims to support the development of measurements within Europe by providing validation of techniques and developing robust protocols to feed into the CEN standards. The project partners will develop facilities to provide simulated area source emissions to enable area source measurement methods to be assessed and to support the development of new techniques. These facilities will themselves be extensions to the state of the art, but in addition they will support and enable the extensions beyond the state of the art of measurement techniques and methods by providing a means to validate new techniques. The test facilities developed will be used to assess the performance of the DIAL technique, TDLAS open path instrumentation and infrared imaging cameras, providing a demonstration of the extent to which they extend the state of the art. A field trial will be used to demonstrate the performance capabilities of these techniques.

\section{Summary}

The EMRP project IMPRESS ("Metrology to underpin future regulation of industrial emissions") [1], which started in 2014, addresses metrology needs in stack emissions monitoring, annual mass emission reporting and area source emission quantification. It aims to enhance the measurement possibilities for emissions of criteria pollutants with lower emission limit values and uncertainties unachievable with current SRMs. In particular, laser-based techniques have reached such a technical maturity that their use in an industrial environment has become realistic. Also developing test facilities within the European NMI community to test and validate methods and to support the development of next generation monitoring methods will be a scope of the project. Furthermore it will develop improved approaches to determining the uncertainties in flow measurement and in annual mass emission values and provide protocols and methodologies for measuring and monitoring emissions from area (including fugitive sources), including the emissions of GHGs. The project will support the establishment of a robust metrology infrastructure to underpin the new monitoring and reporting framework Industry and regulators desperately require.

\section{Acknowledgement}

The EMRP is jointly funded by the EMRP participating countries within EURAMET and the European Union.

\section{References}

1. http://projects.npl.co.uk/impress/

2. Directive $2010 / 75 / \mathrm{EU}$ of the European Parliament and of the Council of 24 November 2010 on industrial emissions (integrated pollution prevention and control). OJ EU L334: 17-119

3. Directive 2000/76/EC of the European Parliament and of the Council of 4 December 2000 on the Incineration of Waste. OJ C L332: 91-111

4. Directive 2001/80/EC of the European Parliament and of the Council of 23 October 2001 on the Limitation of Emissions of Certain Pollutants into the air from Large Combustion Plants. OJ C L309: 121

5. R. Robinson, M. Coleman, M. Williams, R. Elliott, M. Clack, A. Curtis, Validation of an Alternative Method for the Measurement of SO2 Emissions using Instrumental Methods, NPL Report AS26, June 2008

6. EN 14181:2004, Stationary source emissions. Quality assurance of automated measuring systems

7. CEN / TC 264 N 2036, CEN Plenary, 23/24 May 2012, London, UK 
8. Greenhouse Gas Emission Trends (CSI 010), European Environment Agency, 2013

9. 2nd International Workshop on Remote Sensing of Emissions, US EPA, 2008

http://www.epa.gov/ttnchie1/efpac/workshops/remot esens08.html

10. EN 13284-1 (2002) Stationary source emissions Determination of low range mass concentration of dust

11. Williams M (2011) Replacement for Test Report Reference No 114658/QE8601/Particulate PT Round 4. Version 2, September

12. Pogány A, Wager $\mathrm{S}$, Werhahn $\mathrm{O}$, Ebert $\mathrm{V}$, Development and Metrological Characterization of a Tunable Diode Laser Absorption Spectroscopy (TDLAS) Spectrometer for Simultaneous Absolute Measurement of Carbon Dioxide and Water Vapor, Applied Spectroscopy vol. 69(2), 257 (2015) 